
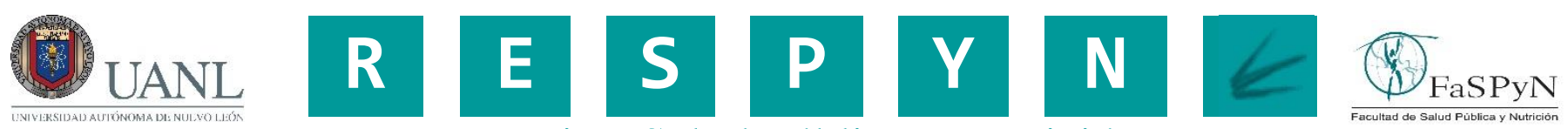

Revista Salud Pública y Nutrición

\title{
MOTIVOS DE LOS ESTUDIANTES DE LA ZONA CENTRO DE MÉXICO PARA ELEGIR LA LICENCIATURA EN NUTRICIÓN.
}

\author{
REASONS OF THE STUDENTS IN THE CENTRAL AREA OF MEXICO TO CHOOSE THE DEGREE IN \\ NUTRITION.
}
Coronel Núñez Samuel*, Pineda Sales Isabel*, Díaz García Rafael*, Reyes Jorge Joel*.
* Universidad Autónoma Metropolitana, México.

Citation: Coronel Núñez S., Pineda Sales I., Díaz García R., Reyes J.J. (2019) Motivos de los estudiantes de la zona centro de México para elegir la licenciatura en nutrición. Revista de Salud Pública y Nutrición, 18 (1), 1-8.

Editor: Esteban G. Ramos Peña, Dr. CS., Universidad Autónoma de Nuevo León, Facultad de Salud Pública y Nutrición, Monterrey Nuevo León, México. Copyright: @2019 Coronel Núñez S. et al. This is an open-access article distributed under the terms of Creative Commons Attribution License [CC BY 4.0], which permits unrestricted use, distribution, and reproduction in any medium, provided the original author and source are credited.

Competing interests: The authors have declared that no competing interests exist.

DOI: https://doi.org/10.29105/respyn18.1-1

Recibido: 31 de octubre 2018; $\quad$ Aceptado: 12 de diciembre 2018

Email: samcor@correo.xoc.uam.mx 


\title{
MOTIVOS DE LOS ESTUDIANTES DE LA ZONA CENTRO DE MÉXICO PARA ELEGIR LA LICENCIATURA EN NUTRICIÓN
}

\author{
Coronel Núñez Samuel*, Pineda Sales Isabel*, Díaz García Rafael*, Reyes Jorge Joel*. \\ * Universidad Autónoma Metropolitana, México.
}

RESUMEN

Introducción. La licenciatura en nutrición en las universidades se inició en México en 1972, hasta el año 2002, se identificaron 32 instituciones y actualmente se estima que existen más de 300 escuelas que ofrecen la licenciatura. Consideramos de la mayor importancia explorar los motivos por los que los estudiantes estudian la licenciatura en nutrición. Objetivo: Analizar los motivos que tuvieron los estudiantes para elegir la licenciatura en nutrición. Material y Métodos: De 2015 a 2018 se aplicaron cuestionarios a 1003 estudiantes que cursaban el primer año y 800 que cursaban el último año de la licenciatura en nutrición en 27 instituciones educativas de la Zona Centro de México: Michoacán, Guanajuato, Hidalgo, Puebla, Morelos, Querétaro, Tlaxcala, Estado de México y la Ciudad de México. Se hicieron dos preguntas, una abierta y otra cerrada a los alumnos del primer año, se solicitó señalar los tres motivos más relevantes, marcando como número uno el de mayor importancia, a los que cursaban el último año solamente se les aplicó la pregunta cerrada. Resultados: Los motivos que se señalaron con mayor frecuencia, tanto por los estudiantes que iniciaban la licenciatura como por los que estaban cercanos a concluirla fueron: a) Interés por trabajar en clínica, b) Orientación vocacional, c) Porque en su familia tuvieron un problema de salud relacionado con la nutrición. En la pregunta cerrada los motivos más importantes fueron: a) Interés por ayudar a la gente, b) El gusto por la carrera, c) Su relación con el área de la salud. Conclusiones: Los motivos para estudiar la licenciatura son muchos y muy diversos, sin embargo, se pueden destacar el interés por trabajar en el área clínica, la orientación vocacional y la posibilidad de ayudar a la gente.

Palabras Clave: Elección de carrera, motivos, licenciatura en nutrición.

\section{ABSTRACT}

Introduction: The bachelor in nutrition began in Mexico in 1972 in the universities. In 2002, 32 institutions were identified and it currently is estimated that there are more than 300 schools that offer the bachelor. We consider important to explore the reasons why students decide to study a Bachelor of nutrition. Objective: Analyze the reasons why students decide to study a Bachelor of nutrition. Methods: They were applied 1003 questionnaires to students that were studying the first year of Bachelor of Nutrition from 2015 to 2018 . Also 800 questionnaires were applied to student of the last year of the Nutrition Bachelor. Students were from 27 educational institutions of the Central Zone of Mexico: Michoacán, Guanajuato, Hidalgo, Puebla, Morelos, Querétaro, Tlaxcala, State of Mexico and Mexico City. Two questions were asked, one open and one closed to the students of the first year, it was requested to mark the three most relevant reasons why they chose the degree, indicating as number one the most important, for those who attended the last year, only the closed question was applied. Results: The reasons were that were pointed out more frequently, both by the students who started the degree and by those who were close to concluding it were: a) Interest in working in the clinical area, b) Vocational orientation, c) Because in their family they had a problem of health related to nutrition. In the closed question the most important reasons were: a) Interest in helping people, b) The taste for the degree, c) Its relationship with the health area. Conclusions: The reasons to study the degree are diverse; nevertheless, we can highlight the interest in working in the clinical area, followed by the vocational direction and the possibility of helping the people.

Key words: Career choice, reasons, degree in nutrition. 


\section{Introducción}

El número de escuelas que ofrecen la licenciatura en nutrición en México se incrementó de manera considerable a partir de 1995, especialmente en la Zona Centro de México (ZCM). Para este estudio consideramos en la ZCM a las siguientes entidades federativas: Michoacán, Guanajuato, Hidalgo, Puebla, Morelos, Querétaro, Tlaxcala, Estado de México y la Ciudad de México.

En México, la licenciatura en nutrición se inició en las universidades en 1972 (Olascoaga, 1977, p.54) y en 1995 se identificaron 20 escuelas que ofrecían la licenciatura, cinco de ellas se ubicaban en la ZCM (AMMFEN,1996, p.15).

En 2015 se identificaron más de 300 escuelas que impartían la licenciatura en el país, de las cuales 102 se ubicaron en la ZCM, lo que significa que a partir del inicio de la licenciatura hasta el año 1995, se fundó una escuela de nutrición en un periodo mayor a cuatro años en promedio y de 1995 a 2015 aproximadamente iniciaron la formación de nutriólogos cuatro escuelas por año.

Dentro de este contexto, consideramos que es de la mayor importancia investigar los motivos por los cuales los estudiantes deciden inscribirse a la licenciatura en nutrición. Se hizo una revisión de la literatura especializada para identificar las investigaciones realizadas en este tema, se encontraron artículos relacionados con las siguientes licenciaturas: pedagogía, (Avendaño y González, 2012, p.21-33), (Geereyat, Cifuentes y Villaroel, 2016, p.1-19); Psicología, Derecho y Biología (Gámez y Marrero, 2003, p.121-131); Área de negocios (Farías, Monforte y García, 2012, p.64-80).

En el caso de Ciencias de la Salud, se encontraron dos artículos, en ambos se reportan los motivos de elección de varias carreras (Verde, Gallardo y Compeán, 2007, p.44-51), (Troncoso, Garay y Sanhueza, 2016, p.55-61). En el primer caso se aplicó un cuestionario a 437 estudiantes de enfermería, nutrición, estomatología y medicina; en el segundo caso se aplicaron entrevistas semiestructuradas a 55 estudiantes de: medicina, nutrición y dietética, enfermería, kinesiología y tecnología médica, de la licenciatura en nutrición solamente participaron diez estudiantes.
En la búsqueda bibliográfica se puso especial atención en las revistas de habla hispana de mayor prestigio en el área de nutrición: Archivos Latinoamericanos de Nutrición, Nutrición Hospitalaria, Revista de Salud Pública y Nutrición y Revista Chilena de Nutrición, en ninguno de los casos se encontraron artículos relacionados con el tema "Motivos de elección de la licenciatura en nutrición".

En la investigación que dio lugar a este documento, se aplicaron cuestionarios a 1803 estudiantes de 27 instituciones educativas de la ZCM pertenecientes a nueve estados de la República Mexicana, 1003 cursaban el primer año de estudios y 800 el último año. El objetivo en este estudio fue analizar los motivos que tuvieron los estudiantes para elegir la licenciatura en nutrición, en el artículo se presentan los resultados de los principales motivos que señalaron los estudiantes y se hicieron comparaciones entre los alumnos del primero y último año, además se comparan los resultados entre hombres y mujeres considerando que la licenciatura desde su creación está integrada en forma mayoritaria por estudiantes del género femenino.

\section{Material y Métodos}

En el período comprendido entre marzo de 2015 y agosto de 2018 se llevó a cabo un estudio transversal observacional de tipo descriptivo (Méndez, 2006, p.12), en las instituciones que ofertan la licenciatura en nutrición en la ZCM que incluyó los estados de: Michoacán, Guanajuato, Hidalgo, Puebla, Morelos, Querétaro, Tlaxcala, Estado de México y la Ciudad de México.

Se incluyó a los alumnos de las Instituciones con las siguientes características:

- 5 años como mínimo de haber iniciado la licenciatura

- Tener al menos una generación de egresados

- Contar con el Reconocimiento de Validez Oficial de Estudios (RVOE) y universidades públicas

- Matrícula superior a 100 estudiantes

El criterio de exclusión fue el siguiente: Instituciones que no otorgaron la autorización para realizar la investigación después haber realizado por lo menos tres intentos por los responsables del estudio. 
La prueba piloto y la validación del cuestionario, que incluye otros aspectos, se hizo mediante su aplicación en dos escuelas públicas y dos privadas.

El tamaño de muestra se obtuvo de la siguiente manera: se identificaron las instituciones que cumplían con los criterios de inclusión y se solicitó a las autoridades correspondientes la autorización para aplicar los cuestionarios a todos los alumnos que cursaban el primero y el último año de estudio, de esta manera, se incluyeron 27 instituciones educativas y se obtuvo una muestra de 1803 alumnos, 800 correspondieron a alumnos que cursaban el último año de estudios, estos se incluyeron porque disponen de más información y criterios de valoración sobre los diferentes aspectos del currículum y la práctica curricular y a 1003 estudiantes del primer año, con el interés de obtener, entre otros aspectos, información sobre los motivos que tuvieron para estudiar la licenciatura en nutrición.

Para la aplicación de cuestionarios, asistieron a cada una de las instituciones educativas por lo menos dos de los integrantes del proyecto y se les explicó a los estudiantes la importancia de la investigación y se les hizo saber que su participación en este estudio era de manera estrictamente voluntaria, por lo que no fue necesario obtener el consentimiento informado por escrito. Para indagar los motivos de elección de la carrera, el cuestionario aplicado a los alumnos de primer año incluyó una pregunta cerrada y una abierta, en tanto que el cuestionario correspondiente a los de último año, solo incluyó la pregunta cerrada.
Para el análisis se utilizó estadística descriptiva y pruebas de chi-cuadrado, con el apoyo de los paquetes Excel 2016 e IMB SPSS Statistics 22.

\section{Resultados}

En este estudio, se analizaron las respuestas de 1803 estudiantes, tanto del primero como del último año, matriculados en 27 Instituciones de la ZCM, de las cuáles $40 \%$ fueron universidades públicas y el $60 \%$ restante fueron universidades privadas.

Datos generales. De los estudiantes de primer año, el $79.4 \%$ correspondieron al sexo femenino y $20.6 \%$ al masculino, en tanto que en los del último año la proporción fue de $84.5 \%$ y de $15.5 \%$ respectivamente. La edad promedio de los primeros fue 20.7 años, con una desviación estándar de 3.81 y de los que estaban por terminar la carrera fue de 24.6 años, con una desviación estándar de 4.03. Con relación al estado civil, el $96 \%$ de los estudiantes de primer año eran solteros, $2 \%$ casados y $2 \%$ tenían otro estado civil, en el caso de los estudiantes de último año, $94 \%$ eran solteros, $5 \%$ casados y $1 \%$ con otro estado civil.

A cada alumno se le solicitaron tres motivos en orden de importancia, el marcado como número uno debía corresponder al motivo más importante. Dentro de los resultados más relevantes se encontró que: a) Interés por el trabajo en clínica, b) Orientación vocacional y c) Porque el estudiante o algún familiar cercano tuvo algún problema relacionado con la nutrición, fueron los tres motivos mencionados con mayor frecuencia. (Tabla 1) 
Tabla 1. Motivos de los estudiantes para elegir la licenciatura en nutrición como carrera profesional

\begin{tabular}{|c|c|c|c|c|c|c|c|c|c|c|c|c|}
\hline \multirow{3}{*}{ MOTIVOS } & \multicolumn{4}{|c|}{$\begin{array}{l}\text { MARCADO COMO } \\
\text { NÚMERO } 1\end{array}$} & \multicolumn{4}{|c|}{$\begin{array}{l}\text { MARCADO COMO } \\
\text { NÚMERO } 2\end{array}$} & \multicolumn{4}{|c|}{$\begin{array}{c}\text { MARCADO COMO NÚMERO } \\
3\end{array}$} \\
\hline & \multicolumn{2}{|c|}{$\begin{array}{l}\text { PRIMER } \\
\text { AÑO }\end{array}$} & \multicolumn{2}{|c|}{$\begin{array}{l}\text { SEGUNDO } \\
\text { AÑO }\end{array}$} & \multicolumn{2}{|c|}{$\begin{array}{l}\text { PRIMER } \\
\text { AÑO }\end{array}$} & \multicolumn{2}{|c|}{$\begin{array}{l}\text { SEGUNDO } \\
\text { AÑO }\end{array}$} & \multicolumn{2}{|c|}{$\begin{array}{l}\text { PRIMER } \\
\text { AÑO }\end{array}$} & \multicolumn{2}{|c|}{$\begin{array}{l}\text { SEGUNDO } \\
\text { AÑO }\end{array}$} \\
\hline & $\mathrm{F}$ & $\%$ & $\mathrm{~F}$ & $\%$ & $\mathrm{~F}$ & $\%$ & $\mathrm{~F}$ & $\%$ & $\mathrm{~F}$ & $\%$ & $\mathrm{~F}$ & $\%$ \\
\hline 1. Influencia de familiares & 57 & 5.7 & 51 & 6.4 & 57 & 5.7 & 48 & 6.0 & 64 & 7.3 & 74.2 & 10.3 \\
\hline 2. Perspectiva de obtener altos ingresos & 40 & 4 & 25 & 3.1 & 60 & 6.0 & 55 & 6.9 & 88 & 10 & 74.2 & 10.3 \\
\hline 3. Orientación vocacional & 191 & 19 & 178 & 22.3 & 142 & 14.2 & 159 & 19.9 & 108 & 12.3 & 105 & 14.6 \\
\hline 4. Influencia de amigos & 5 & 0.5 & 8 & 1 & 8 & 0.8 & 13 & 1.6 & 7 & 0.8 & 17.3 & 2.4 \\
\hline 5. Para lograr prestigio social & 32 & 3.2 & 6 & 0.8 & 39 & 3.9 & 20 & 2.5 & 57 & 6.5 & 31 & 4.3 \\
\hline 6. Interés por el trabajo en clínica & 328 & 32.7 & 296 & 37 & 212 & 21.1 & 206 & 25.8 & 91 & 10.3 & 75.6 & 10.5 \\
\hline 7. Por tener experiencia ocupacional previa & 10 & 1 & 11 & 1.4 & 32 & 3.2 & 17 & 2.1 & 27 & 3.1 & 28.8 & 4 \\
\hline $\begin{array}{l}\text { 8. Por considerar que al concluir sería fácil } \\
\text { encontrar trabajo }\end{array}$ & 19 & 1.9 & 9 & 1.1 & 46 & 4.6 & 34 & 4.3 & 55 & 6.3 & 46.1 & 6.4 \\
\hline 9. Por mejorar tu imagen corporal & 42 & 4.2 & 20 & 2.5 & 93 & 9.3 & 56 & 7.0 & 100 & 11.4 & 86.4 & 12 \\
\hline 10. Por asistir a un gimnasio & 33 & 3.3 & 12 & 1.5 & 59 & 5.9 & 18 & 2.3 & 50 & 5.7 & 18 & 2.5 \\
\hline $\begin{array}{l}\text { 11. Porque tu o algún familiar cercano tuvo } \\
\text { algún problema relacionado con la nutrición }\end{array}$ & 155 & 15.5 & 106 & 13.3 & 142 & 14.2 & 117 & 14.6 & 135 & 15.3 & 108 & 15 \\
\hline 12. Otros & 91 & 9.1 & 78 & 9.8 & 113 & 11.3 & 57 & 7.1 & 97 & 11 & 55.4 & 7.7 \\
\hline TOTAL & 1003 & 100 & 800 & 100 & 1003 & 100 & 800 & 100 & 880 & 100 & 720 & 100 \\
\hline
\end{tabular}

Al comparar por sexo sobre los motivos que tienen los estudiantes para estudiar a licenciatura (se analizó solamente el motivo más importante), se observó que: a) Interés por el trabajo en clínica fue marcado por $36.7 \%$ de las mujeres y $26.3 \%$ de los hombres, b) Orientación vocacional por $20.7 \%$ de las mujeres y $19.7 \%$ de los hombres y c) Porque el estudiante o algún familiar cercano tuvo algún problema relacionado con la nutrición por $14.6 \%$ y $14.0 \%$. (Tabla 2)

Tabla 2. Motivos de los estudiantes para elegir la licenciatura en nutrición con relación al sexo.

\begin{tabular}{|c|c|c|c|c|}
\hline \multirow{2}{*}{ Motivos } & \multicolumn{2}{|c|}{ Mujeres } & \multicolumn{2}{|c|}{ Hombres } \\
\hline & $\mathrm{F}$ & $\%$ & $\mathrm{~F}$ & $\%$ \\
\hline 1. Influencia de familiares & 76 & 5.3 & 32 & 8.8 \\
\hline 2. Perspectiva de obtener altos ingresos & 41 & 2.9 & 24 & 6.6 \\
\hline 3. Orientación vocacional & 297 & 21 & 72 & 20 \\
\hline 4. Influencia de amigos & 8 & 0.6 & 5 & 1.4 \\
\hline 5. Para lograr prestigio social & 26 & 1.8 & 12 & 3.3 \\
\hline 6. Interés por el trabajo en clínica & 528 & 37 & 96 & 26 \\
\hline 7. Por tener experiencia ocupacional previa & 11 & 0.8 & 10 & 2.7 \\
\hline $\begin{array}{l}\text { 8. Por considerar que al concluir sería fácil encontrar } \\
\text { trabajo }\end{array}$ & 22 & 1.5 & 6 & 1.6 \\
\hline 9. Por mejorar tu imagen corporal & 44 & 3.1 & 18 & 4.9 \\
\hline 10. Por asistir a un gimnasio & 31 & 2.2 & 14 & 3.8 \\
\hline $\begin{array}{l}\text { 11. Porque tu o algún familiar cercano tuvo algún } \\
\text { problema relacionado con la nutrición }\end{array}$ & 210 & 15 & 51 & 14 \\
\hline 12. Otros & 144 & 10 & 25 & 6.8 \\
\hline
\end{tabular}

La mayor parte de resultados fueron semejantes entre hombres y mujeres, solamente se encontraron

diferencias significativas a valores de $\mathrm{p}<0.05$, con relación al interés por trabajar en nutrición clínica con un porcentaje mayor de mujeres que de hombres y en el caso de la perspectiva de obtener altos ingresos, fue mayor el porcentaje de hombres que de mujeres.

Para conocer si los estudiantes tuvieron otros motivos -que no fueron incluidos en la pregunta cerrada- para la elección de la carrera, se hizo la misma pregunta, pero de forma abierta a estudiantes de primer año, se categorizaron las respuestas y se encontró que el $16.3 \%$ de los estudiantes decidieron estudiar la licenciatura porque tuvieron interés o gusto por la carrera, el segundo motivo mencionado fue "por ayudar a la gente" (12.6\%) y el tercero, con un porcentaje similar, fue la relación de la nutrición con el área de la salud (12\%). Fueron mencionados otros motivos, con porcentajes menores al 5\% que pueden revisarse en la tabla 3 . Se puede observar que las respuestas entre la pregunta abierta y la cerrada son similares, a excepción de "ayudar a la gente" que no se contempló en la pregunta cerrada. 
Tabla 3. Motivos de los estudiantes para elegir la licenciatura en nutrición (pregunta abierta)

\begin{tabular}{lccc}
\hline \multicolumn{1}{c}{ Motivos } & \multicolumn{2}{c}{ MOTIVO } \\
\cline { 3 - 4 } \cline { 3 - 4 } \cline { 4 - 4 } 1. Interés o gusto por la carrera & F & $\%$ \\
\hline 2. Por ayudar a la gente & & 163 & 16.3 \\
3. La relación de la nutrición con el área de la salud & & 126 & 12.6 \\
4. Nuevos conocimientos acerca del metabolismo con & & 12 \\
base en una alimentación correcta & 48 & 4.8 \\
5. Influencia familiar & 45 & 4.5 \\
6.Problemática de salud actual en México & 40 & 4 \\
7.Enfermedades asociadas & 37 & 3.7 \\
8. Importancia de la carrera & 35 & 3.5 \\
9. Prevención de enfermedades & 30 & 3 \\
10. Otros motivos & 359 & 35.8 \\
\hline & TOTAL & 1003 & 100
\end{tabular}

\section{Discusión}

El motivo más importante marcado como número uno, tanto por los alumnos de primer año como del último, fue: "Interés por trabajar en clínica". Este motivo fue señalado en el primer caso por el $32.7 \%$ de los alumnos y en el segundo caso por el $37 \%$; en el porcentaje acumulado, es decir, el que representa el total de alumnos que manifestaron este motivo, ya sea en primero, segundo o tercer lugar, fue para los alumnos del primer año $64.1 \%$ y para los del último año el $73.3 \%$.

El interés por trabajar en nutrición clínica ha sido una constante en los estudiantes de las universidades mexicanas, en una investigación a nivel nacional, se mostró que el $54.3 \%$ de los estudiantes de primer año y el $57.7 \%$ del último año manifestaron interés por trabajar en el campo de Nutrición Clínica (Coronel y Díaz, 2015, p.12), lo que significa que el campo profesional de la nutrición clínica fue superior a la suma de los otros tres campos definidos por Asociación Mexicana de Miembros de Facultades y Escuelas de Nutrición (Nutrición poblacional, Servicios de alimentos y Tecnología alimentaria). Esta situación se manifestó también en la ubicación de los egresados en el campo laboral, en 1996, el $43 \%$ de egresados trabajaba en el área nutrición clínica (AMMFEN, 1996, p.52) y en 2006, el porcentaje se incrementó al 59\% (AMMFEN, 2006, p.64), la situación anterior es diferente a otros países como Chile, que en 1992 el 25.1\% se ubicó en Nutrición clínica (Olivares, Soto y Aguayo, 1992, p.52) y en 2013, solamente el 12.9\% (González, 2013, p.11).

Entre los posibles factores que pueden explicar los altos porcentajes en el campo de nutrición clínica para el caso de México se encuentran los siguientes: a) La enseñanza de la nutriología se inició en los hospitales, lo cual incidió significativamente en el desarrollo de la profesión, ya que la práctica profesional dominante guarda relación con su origen, b) Una cantidad importante de programas de licenciatura se fundaron asociados a facultades $o$ escuelas de medicina, lo que posiblemente influyó en un enfoque dirigido a nutrición clínica. (Coronel y Díaz, 2015, p.7)

El segundo motivo en orden de importancia fue "orientación vocacional", este término ha tenido diferentes concepciones y su origen se remonta a principios del siglo pasado (Gavilán, 2006, p.32), y ha tomado una gran variedad de definiciones y se han tenido diferentes objetivos, se señala que la orientación vocacional se ocupa de que los estudiantes no se equivoquen cuando tomen una decisión vocacional y así poder evitar fracasos en sus vidas; también se considera que los estudiantes tomen las mejores decisiones vocacionales, si no la correcta, por lo menos la más adecuada, atendiendo a sus características de personalidad e interés detectados (Oliveros y González, 2012, p.128). En varios artículos se argumenta que una de las principales funciones de la orientación vocacional, es presentar la información existente en varias universidades para que el estudiante seleccione la que más le guste.

En este estudio, el $19 \%$ de los estudiantes del primer año y el $19.9 \%$ del último año señalaron a la "orientación vocacional" como el segundo motivo más importante marcado como número 1 y en el porcentaje acumulado lo mencionaron el $45.5 \%$ y el $54.5 \%$ respectivamente. En algunas investigaciones la orientación vocacional se ha considerado como un factor determinante, por ejemplo, para estudiar medicina (Machado y otros, 2013, p.183), sin embargo, en otros estudios tuvo resultados controvertidos, la mayoría de los estudiantes de las carreras de kinesiología, nutrición y dietética y enfermería identificaron la limitada o nula orientación recibida desde los establecimientos educacionales para la selección de su carrera universitaria.

El tercer motivo señalado en orden de importancia fue relacionado con la situación de que el estudiante o algún familiar cercano tuvo algún problema relacionado con la nutrición, mencionado por el 
$15.5 \%$ de los alumnos del primer año y por el $13.3 \%$ de los alumnos del último año. Con relación al porcentaje acumulado, el $45 \%$ de los alumnos que iniciaba la carrera y $42.9 \%$ de los que estaban cercanos a concluirla marcaron este motivo.

Aunque el motivo fue relacionado con problemas personales o familiares de nutrición, consideramos que es solo un reflejo de la situación epidemiológica que afecta a todo el país, como se reporta en la Encuesta Nacional de Salud y Nutrición de Medio Camino 2016, en la que se establece que, en el caso de las mujeres de 10 a 49 años, en el periodo de 1988 a 2016, el sobrepeso se incrementó en un $42.4 \%$ y la prevalencia de obesidad en $290.5 \%$. En el caso de los hombres el periodo de seguimiento es más corto, de 2000 a 2016, durante este periodo la prevalencia de sobrepeso aumentó $1.1 \%$ y la obesidad se incrementó en $42.8 \%$, en lo que se refiere a Diabetes, enfermedad íntimamente relacionada con la nutrición, paso de $5.8 \%$ en el año 2000 a $9.4 \%$ en el año 2016 (ENSANUTMC,2016, p.46-80).

Es probable que la situación epidemiológica de México sea uno de los motivos por lo que muchos estudiantes decidieron elegir la licenciatura en nutrición, en este sentido, es importante destacar que actualmente más de la tercera parte de los egresados se dedican a la consulta privada, ya sea como trabajo principal o secundario y que la obesidad y la diabetes son las principales causas por las que los pacientes acuden a recibir atención nutriológica (AMMFEN, 2015, p.124).

Con una frecuencia mucho menor que los motivos anteriores, el cuarto motivo en orden de importancia fue: "Influencia de familiares", marcado como número uno por el $5.7 \%$ de los estudiantes del primer año y $6.4 \%$ de los del último, la influencia de la familia no se identificó como un factor que haya motivado la elección de la carrera en la gran mayoría de los estudiantes encuestados. En otros estudios esta misma tendencia se presentó en las carreras de enfermería, nutrición y dietética, kinesiología y tecnología médica, sin embargo, sí constituyó un motivo importante para estudiantes de medicina (Troncoso, Garay y Sanhueza, 2016, p.3). La reducida influencia familiar también se encontró en otras investigaciones (Peinado y Fernández, 2011, p.392); (De la mano y Moro, 2010, p.212).
Otros motivos que, aunque no fueron mencionados con tanta frecuencia como los señalados previamente son: "Por mejorar tu imagen corporal" y "Por asistir a un gimnasio". Para los profesores de la licenciatura en nutrición, es frecuente encontrar en las aulas uno o más estudiantes que practican fisicoculturismo o algún deporte de manera constante, es posible que este grupo haya accedido a la licenciatura en nutrición por su interés en el área de nutrición y deporte. Es conveniente señalar que, en el año 2007, cuando la AMMFEN actualizó los campos profesionales se propuso incluir como un nuevo campo "nutrición y deporte", sin embargo, no se aceptó, actualmente existen mayores evidencias para que la AMMFEN reconsidere su inclusión (Coronel y Díaz, 2015, p.3).

Otro motivo que fue mencionado con una frecuencia considerable fue "la perspectiva de obtener altos ingresos", que fue marcada como número uno por el $4.0 \%$ de los alumnos del primer año y $3.1 \%$ del último año y en el acumulado alcanzó un porcentaje superior al $20 \%$ en ambos casos. En este sentido es conveniente comentar que en el segundo estudio nacional de egresados realizado por la AMMFEN se señaló, que uno de los mayores problemas de la profesión se refiere a los bajos salarios que se paga a los egresados y que en promedio es inferior comparado con el salario que se paga a otros profesionales de la salud como médicos, dentistas y químicos (AMMFEN, 2006, p.61).

Los motivos que tuvieron frecuencias marginales fueron: a) Influencia de amigos, b) Por considerar que al concluir sus estudios sería fácil encontrar trabajo, c) Por experiencia ocupacional previa. En los tres casos, fueron marcados como número uno por un porcentaje inferior al $2 \%$ tanto por los alumnos del primero como del último año.

En esta investigación se propuso hacer la comparación de resultados con respecto al sexo, considerando que la población femenina es ampliamente mayoritaria en la licenciatura, sin que se tengas suficientes elementos para justificar esta situación. En 1996, la AMMFEN reportó que el 87\% de egresados correspondió al sexo femenino y el $13 \%$ al masculino y después de una amplia discusión, dentro del grupo de trabajo, se concluyó que "las instituciones que ofrecen la licenciatura no restringen el acceso a los hombres y que los diferentes campos 
profesionales pueden ser desarrollados indistintamente con el mismo nivel de calidad" (AMMFEN, 1996, p.38). La proporción de hombres se ha incrementado con el tiempo, $82.6 \%$ mujeres y $17.4 \%$ hombres (AMMFEN, 2006, p.44) que es una distribución semejante a la encontrada en este estudio.

De acuerdo a los resultados, se puede observar que son similares en nueve de las once opciones y que solamente en "interés para trabajar en clínica" y "perspectivas de alto ingreso", de acuerdo con la prueba estadística de chi-cuadrado existen diferencias significativas a valores de $\mathrm{p}<\mathrm{a} 0.05$.

En lo que respecta a la mayor proporción de mujeres que se interesa por trabajar en nutrición clínica es difícil explicar la diferencia porque existen múltiples variables para esta situación, sin embargo, es probable que una de las razones, sea la posibilidad de trabajar en la consulta privada que actualmente representa una de las fuentes más importantes para el empleo, y que permite flexibilidad de horarios, que fue uno de los motivos más importantes señalados por los nutriólogos que trabajan en esta área (AMMFEN, 2015, p.111).

En lo que se refiere a la perspectiva de obtener altos ingresos, la proporción de hombres fue mayor que la de las mujeres, este es un aspecto que se ha estudiado más y se explica en función del peso de la socialización diferencial respecto de los roles esperados en función del género, que considera al hombre como el principal sostén del hogar, asignando al salario femenino una función de apoyo (Quattrocchi y otros, 2017, p.33).

Dentro de las respuestas obtenidas de la pregunta abierta que solamente se aplicó a los alumnos del primer año, solo se consideraron las que tenían una frecuencia superior al $3 \%$ y es importante señalar que se obtuvo una amplia diversidad de respuestas con frecuencias menores y que se engloban en la opción otros con el $35.8 \%$.

En las respuestas que se obtuvieron con una frecuencia mayor al $3 \%$ podemos observar que hay similitud en los aspectos referentes a: influencia familiar, problemática de la salud en México y enfermedades asociadas, sin embargo, consideramos de la mayor importancia destacar que las dos respuestas con mayor frecuencia fueron: "interés o gusto por la carrera" con el $16.3 \%$ y "ayudar a la gente" con el 12.6\%. En el primer caso consideramos que es difícil hacer observaciones ya que el gusto puede estar relacionado con múltiples variables; en el segundo caso, resulta agradable que, en una pregunta abierta, sea la segunda que obtuvo la mayor frecuencia, tomando como base que la ayuda a la gente, particularmente a la más vulnerable es una obligación moral de las universidades y en especial de las profesiones de la salud.

Los egresados de la licenciatura en nutrición pueden ayudar a muchas personas en cualquiera de los campos profesionales, especialmente en el campo profesional denominado "nutrición poblacional" antes "nutrición comunitaria" si consideramos que las enfermedades relacionadas con la nutrición afectan a millones de personas y que en este campo se puede actuar con grupos de población y particularmente a nivel preventivo.

\section{Conclusiones}

Los motivos de los estudiantes de la Zona Centro de México para elegir la licenciatura en nutrición son muy diversos, sin embargo, se pueden destacar: a) El interés por trabajar en nutrición clínica, b) La orientación vocacional, c) El panorama epidemiológico de la salud en México relacionado con la nutrición y d) La posibilidad de ayudar a la gente.

No se encontraron diferencias importantes entre las respuestas de los alumnos que están iniciando la licenciatura con relación a los que están por terminarla. Con respecto al género, las respuestas también fueron similares, excepto en dos casos, en las que hubo diferencias estadísticamente significativas, que fue lo relacionado con el trabajo en nutrición clínica y las posibilidades de obtener altos ingresos.

De acuerdo con la revisión en la literatura especializada, se puede afirmar que este tema ha sido poco estudiado y que es de mucha relevancia, considerando el acelerado incremento de la matrícula en el país. Es conveniente que las instituciones educativas que ofrecen la licenciatura otorguen información sobre el plan de estudios y el mercado laboral a sus estudiantes antes del ingreso o durante el primer año de la carrera. 


\section{Agradecimientos}

Agradecemos al Programa para el Desarrollo Profesional Docente (PRODEP) por el apoyo brindado al Cuerpo Académico "Investigación Educativa en las licenciaturas de Ciencias Biológicas y de la Salud", también agradecemos el apoyo de las 27 Instituciones participantes en este estudio.

\section{Bibliografía}

AMMFEN. (1996). Los nutriólogos en México: seguimiento de egresados. Ciudad de México, México: Trillas.

AMMFEN. (2006). Los nutriólogos en México: un estudio de mercado laboral. Ciudad de México, México: Trillas.

AMMFEN. (2015). Los nutriólogos y la consulta privada. México: Trillas.

Avendaño, C. y González, R. (2012). Motivos para ingresar a las carreras de Pedagogía de los estudiantes de primer año de la Universidad de Concepción. Estudios Pedagógicos. 38(2),21-33.

Coronel, S. y Díaz, R. (2015). Formación, mercado laboral y necesidades sociales en el campo de la nutrición comunitaria. En, J.L. Castillo (Ed.), Nutrición Comunitaria (pp.1-21). México: ELSEVIER.

De la mano, M., Moro, M. y Da Graça, M. (2010). Motivaciones en la elección de la carrera universitaria: Metas y objetivos de los estudiantes de la Facultad de Traducción y Documentación de la Universidad de Salamanca. Ediciones Universidad de Salamanca. 210.

Instituto Nacional de Salud Pública. (2016). Encuesta Nacional de Salud y Nutrición de Medio Camino. México.

Farías, M.G., Monforte, G. y García, M. (2012). Criterios, percepciones y personalidad de los estudiantes que determinan la elección de una carrera profesional en el área de negocios. Revista Iberoamericana de Educación Superior. 7(19), 64-80.

Gavilán, M. (2006). La transformación de la Orientación Vocacional. Homosapiens Ediciones. Argentina.

Gámez, E. y Marrero, H. (2003). Metas y motivos en la elección de la carrera universitaria: Un estudio comparativo entre psicología, derecho y biología. Anales de psicología. 19(1),121-131.
Geereyat, O., Cifuentes y Villaroel, M.C. (2016). Factores que inciden en las condiciones de empleabilidad de los egresados de pedagogía. Revista Actualidades Investigativas en Educación. 16(1),1-19.

González, M.A. (2013). Mercado Laboral del Nutricionista en Chile. Revista del Colegio de Nutricionistas. 9(1), 6-11.

Machado, B., Llerena, M., Mesa, L., Quintero, M. y Miñoso, I. (2013) La orientación vocacional: factor determinante en la decisión de estudiar Medicina. Revista Educación Médica del Centro. 5(3), 96-183.

Méndez, I. (2006). Protocolo de Investigación. México: Trillas.

Olascoaga, Q. (1977). Historia de la nutriología. México: ISSSTE.

Olivares, S., Soto, D., Zacarias, I. y Aguayo, M. (1992). Estudio del perfil profesional del Nutricionista en Chile. Archivos Latinoamericanos de Nutrición. 42(2 supl), 48-58.

Oliveros, O. y González, J. (2012). Hacia un nuevo paradigma en Orientación Vocacional. Paradigma. $33(2), 127-141$.

Peinado, F. y Fernández, M. (2011). Reflexión sobre la motivación de los alumnos de grado en la elección de estudios de comunicación en las universidades de Madrid. Estudios sobre el mensaje periodístico. 17(2), 383-400.

Quatrocchi, P., Flores, C., Cassullo, G., Moulia, L., Marco, M., Shafferstein C., Pereda, Y. y Siniuk, D. (2017). Motivación y género en la elección de la carrera. Revista de Educación y Desarrollo. (41), 27-35.

Troncoso, C., Garay, B. y Sanhueza, P. (2016). Percepción de las motivaciones en el ingreso a una carrera del área de la salud. Horizonte Médico. 16(1), 55-61.

Verde, E., Gallardo, G. y Compeán, S. (2007). Motivos de la elección de la carrera en mujeres estudiantes de profesiones de la salud. Educación Médica. 10(1), 44 51. 